Nota técnica

Volumen 30(3):871-884. Septiembre-diciembre, 2019

e-ISSN 2215-3608, doi:10.15517/am.v30i3.36931

http://www.revistas.ucr.ac.cr/index.php/agromeso

\title{
Caracterización técnica y económica de la producción de carne ovina en Córdoba, Colombia $^{1}$
}

\section{Technical and economic characterization of lamb meat production in Córdoba, Colombia}

\author{
Lorena Inés Mestra-Vargas ${ }^{2}$,Antonio María Martínez-Reina ${ }^{2}$, Martha Oliva Santana-Rodríguez ${ }^{2}$
}

1 Recepción: 29 de abril, 2019. Aceptación: 12 de junio, 2019. Este trabajo formó parte del proyecto de investigación de la Corporación Colombiana de Investigación Agropecuaria (Agrosavia): 1010-0, financiado por el Ministerio de Agricultura y Desarrollo Rural (MADR), Colombia.

2 Corporación Colombiana de Investigación Agropecuaria (AGROSAVIA). Km 13 vía Montería - Cereté, Córdoba, Colombia. Teléfono: 0914227300.1mestra@agrosavia.co (autor para correspondencia; https://orcid.org/0000-0003-3717-0153), amartinezr@agrosavia.co (https:// orcid.org/0000-0002-9312-842X), msantana@agrosavia.co (https://orcid.org/0000-0002-0248-9298).

\section{Resumen}

Introducción. El conocimiento de las variables biofísicas, tecnológicas y económicas de los sistemas ovinos, representan elementos de valor para la caracterización y el análisis productivo de la especie. Objetivo. El objetivo de este trabajo fue caracterizar técnica y económicamente, el sistema de producción de ovinos de carne en el departamento de Córdoba, Colombia, con el fin de generar una línea de base que permita comparar las opciones tecnológicas con la tecnología local. Materiales y métodos. La información se obtuvo del trabajo realizado en cuatro unidades productivas con 108 ovinos machos de manejo semi-intensivo. Los datos se obtuvieron entre enero y septiembre de 2018. Como instrumento de información se usó la encuesta estructurada, se aplicaron técnicas estadísticas como medidas de tendencia central y de dispersión, para el análisis económico se usó un patrón de costos para cada unidad productiva y el cálculo de los indicadores de retorno como la tasa interna de retorno y valor presente neto. Los coeficientes técnicos se analizaron económicamente. Resultados. Las ganancias diarias de peso fueron de 80-132 g día ${ }^{-1}$, con peso

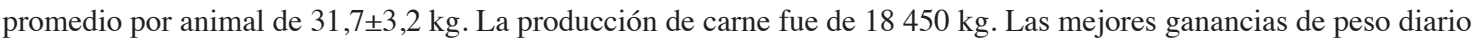
$(\mathrm{p}<0,0001)$ y peso final, se observaron en la finca Campanario con 33,4 $\pm 4,1$ como respuesta al manejo técnico que generaron mayores unidades productivas y por ende, mayores ingresos. El costo unitario promedio fue de USD \$1,059 $\mathrm{kg}^{-1}$ la rentabilidad de $28 \%$, y la tasa interna de retorno de 4,5\%. Conclusión. Los sistemas de producción evaluados fueron rentables con capacidad de recuperar la inversión. El análisis de los márgenes de comercialización mostró la inequidad en la participación del productor en el valor que paga el consumidor por el producto, ya que recibe solo 18 centavos por cada unidad monetaria pagada, el resto se capta en la intermediación.

Palabras clave: ovinos, sistemas de explotación, indicadores económicos, comercialización.

\begin{abstract}
Introduction. The knowledge of the biophysical, technological, and economic variables of lamb production systems represents valuable elements to delve into the productive characterization and analysis of the species. Objective. The aim of this study was to characterize technically and economically, the lamb meat production system


in the department of Córdoba, Colombia, to generate a baseline that allows comparing technological options with local technology. Materials and methods. The information was obtained from a fieldwork carried out in four productive units with 108 male lambs under semi-extensive management. The data were obteined between January to September 2018. Structured surveys, as well as statistical techniques were applied, including central tendency and dispersion measures. For the economic analysis a cost pattern was used for each productive unit and the calculation of return indicators such as the internal rate of return and the net present value. The technical coefficients were analyzed economically. Results. The daily weight gains were $80-132 \mathrm{~g} \mathrm{day}^{-1}$, with an average weight per animal of $31.7 \pm 3.2$ $\mathrm{kg}$. Meat production was $18.450 \mathrm{~kg}$. The best gains of daily weight $(\mathrm{p}<0.0001)$ and final weight were observed in the Campanario farm with $33.47 \pm 4.1 \mathrm{~kg}$ as a response to the technical management that generated greater productive units and higher incomes. The average unit cost was USD $\$ 1.05 \mathrm{~kg}^{-1}$, the profitability of $28 \%$, and the internal rate of return was $4.5 \%$. Conclusions. The production systems evaluated were profitable with the capacity to recover the investment. The analysis of marketing margins showed the inequality in the producers' participation in the value paid by the consumer for the product since they receives only 18 cents for each monetary unit paid the rest is captured by the intermediation.

Keywords: sheep, farming systems, economic indicators, commercialization.

\section{Introducción}

La producción ovina en Colombia es una actividad relevante, la cual registra aumento en las exportaciones a una tasa del $10 \%$, principalmente hacia las Antillas, donde de acuerdo con datos del Ministerio de Agricultura y Desarrollo Rural (MADR, 2018) en el año 2018 se exportaron 56 toneladas de carne, igualmente esta actividad reviste importancia en el consumo interno en los hogares, que también presentó tasas de crecimiento del 10,1\% en el periodo comprendido entre el año 2010 al año 2017 (MADR, 2018). Esto ha propiciado un ambiente favorable para que los inventarios crezcan con tasas del $40 \%$ que corresponde a un número total de ovinos de 1400000 cabezas de los cuales la Región Caribe representa el 69 \%, y el departamento de Córdoba el $6 \%$ (Espinal et al., 2006; MADR, 2018).

Generalmente las unidades productivas son pequeñas, manejadas bajo un sistema de producción semi intensivo y con bajo nivel tecnológico. Con relación a la contribución de la cadena sobre la productividad nacional, se ha observado una tendencia al incremento en el número de animales en producción sacrificados con destino a la producción de carne, así en el año 2010 se sacrificaron 147500 cabezas, para pasar a un número de 211231 cabezas en el año 2017, con producciones de carne de 2138 toneladas en 2013 y 3063 en 2017, lo cual representa un incremento de producción de carne promedio del 1,02\% (FAOSTAT, 2017).

En la región Caribe colombiana, actualmente existe una baja competitividad en los sistemas de producción de ovinos, debido a que este sector no ha sido sustentado académicamente, lo que no permite identificar los nichos productivos desde las diferentes ecorregiones, para cuantificar la demanda de mercados nacionales e internacionales de los productos y subproductos generados en los sistemas de producción.

El departamento de Córdoba en el año 2016, representó el 6 \% del total de producción de carne ovina a nivel nacional. Este crecimiento en el inventario y el consumo per cápita del ovino impulsa la necesidad de generar ventajas competitivas que lleven a la ovinocultura de la Región Caribe por el camino de la competitividad, aprovechando las oportunidades en el mercado nacional y de exportación (Garnier, 2010).

En los sistemas de ovinos del departamento de Córdoba aún se desconoce la dinámica del manejo técnico para la producción de carne de una manera más eficiente. Según Moreno y Grajales (2017), a nivel nacional, los mayores controles productivos son realizados en los sistemas de producción de genética y de carne con mayor grado de especialización. Estudios realizados por Vega-Pérez (2017) para la caracterización por tamaño (número de 
cabezas) de las unidades productivas de cuatro zonas geográficas colombianas, encontró que en el departamento de Córdoba, más del $70 \%$ de las unidades productivas presentan inventarios superiores a los cincuenta animales por unidad, y afirma que actualmente no existen estudios suficientes relacionados con la productividad de la especie, y que existen severas deficiencias por falta de información, diferencias en el tamaño de las granjas, la calidad de la tierra o la productividad del trabajo.

Uno de los principales atributos de los sistemas de producción ovina es su gran adaptación a los terrenos, por ser animales de peso liviano y talla pequeña en comparación con grandes rumiantes, con pocas posibilidades de compactación de los suelos y a la vez, por su capacidad de adaptación a condiciones climáticas adversas, de topografía o de terreno (Acero-Plazas, 2014). Los sistemas de producción ovinos son empresas familiares ubicadas con frecuencia en las zonas menos favorecidas y ofrecen ventajas ambientales, socioeconómicas o nutricionales (Zervas y Tsiplakou, 2011). Esta actividad se ha constituido en una fuente de alimento y una forma natural de subsistencia para el habitante de la zona rural, contribuyendo a la seguridad alimentaria.

La investigación biofísica en sistemas de producción animal, en particular los ovinos para la producción de carne con destino a los mercados locales, nacionales e internacionales, requiere de una caracterización tecnológica y socioeconómica. Esto podría permitir conocer la tecnología local de producción utilizada, para después comparar las opciones tecnológicas que permitan a futuro medir sus bondades desde el punto de vista económico, y de esta forma adentrarse a la medición de la adopción de las tecnologías, que no es más que su incorporación al sistema de producción. La información debe partir del productor, quien es el principio y fin del proceso de generación de tecnologías por considerarse el beneficiario directo del conocimiento científico, y quien finalmente toma las decisiones de incorporar o no los productos tecnológicos que genere la investigación biofísica al sistema de producción (CIMMYT, 1998).

Sobre la caracterización de sistemas de producción en ovinos se han realizado algunos trabajos entre los que se citan el de Freire et al. (2013), cuyo objetivo fue analizar seis sistemas de producción mediante entrevistas semiestructuradas, análisis de variables biofísicas y tecnologías de producción y análisis de costos por componentes y márgenes brutos; el trabajo concluyó que esta actividad presenta márgenes brutos positivos y, en algunos casos, superiores a los sistemas de ganadería bovina.

En un análisis de la comercialización de ovinos de pelo en México, se aplicó una encuesta a veintiséis productores, y se logró identificar canales y márgenes de comercialización, concluyendo que el mayor porcentaje (79 \%) de agentes económicos que intervienen en la comercialización de los ovinos son intermediarios, ya que solo el $21 \%$ vende directamente al consumidor final (Hernández-Martínez et al., 2013). En la misma investigación también se observó que el productor solo participa con el 17,75\% del precio que paga el consumidor, y los intermediarios y el acopiador de ovinos en pie captan el 82,24\%.

El objetivo de este trabajo fue caracterizar técnica y económicamente, el sistema de producción de ovinos para carne en el departamento de Córdoba, con el fin de generar una línea de base que permita comparar las opciones tecnológicas con la tecnología local.

\section{Materiales y métodos}

El trabajo constó de dos partes, una donde se hizo la caracterización técnica, con base en el manejo del sistema de producción con la tecnología local de producción donde se destacan los parámetros productivos, y la otra parte, se realizó un análisis económico de estas tecnologías que se complementaron con el estudio de los márgenes de comercialización y la participación directa del productor como una manera de adentrarse al análisis de los encadenamientos productivos. 


\section{Localización del estudio}

El marco geográfico de esta actividad fueron las zonas productoras de ovinos con destino a carne en el departamento de Córdoba. La información se obtuvo del trabajo de campo realizado entre los meses de enero a septiembre de 2018. Para la definición de las unidades productivas donde se obtuvo la información se partió de la base de datos de los productores adscritos a la Asociación Local y Nacional de Ovinocultores de Córdoba (ASOVICOR), con el objeto de identificar y localizar el número de fincas por municipio. El instrumento utilizado fue una encuesta estructurada con preguntas agrupadas en cuatro categorías; tamaño de las unidades productivas, finalidad de la producción, manejo animal e indicadores económicos y márgenes de comercialización. En total se aplicaron veintiseis encuestas en las unidades productivas (UP) o fincas dedicadas a la cría de ovinos, se usó como modelo la metodología de la Cartografía Mundial de los Sistemas de Alimentación Animal (FAO, 2014). Posterior a la aplicación de las encuestas, se seleccionaron cuatro unidades productivas según los siguientes criterios: i) disponibilidad y actitud del productor para suministrar la información. ii) legalidad de la tenencia de la tierra de la unidad productiva y iii) cumplimiento de las condiciones necesarias para desarrollar el estudio: zona de fácil acceso vial y presencia de personal encargado responsable permanente en la unidad productiva.

Las unidades productivas seleccionadas se localizan en las subregiones de San Jorge, Sabanas, Medio Sinú y Bajo Sinú, como se describe en el Cuadro 1.

Cuadro 1. Localización de las unidades productivas de corderos y número de animales en Córdoba, Colombia. 2018.

Table 1. Location of the productive unit of lambs and number of animals in Cordoba, Colombia. 2018.

\begin{tabular}{|c|c|c|c|c|}
\hline Municipios & Subregión de Córdoba & Unidad productiva & Localización & ${ }^{1}$ Número de animales \\
\hline Planeta Rica & San Jorge & Campanario & $\begin{array}{l}\text { Longitud: } 08^{\circ} 22^{\prime} 33,9^{\prime \prime} \\
\text { Latitud: } 075^{\circ} 34^{\prime} 11,6^{\prime \prime}\end{array}$ & 36 \\
\hline Sahagún & Sabana & La Esperanza & $\begin{array}{l}\text { Longitud: } 08^{\circ} 57^{\prime} 27,7^{\prime \prime} \\
\text { Latitud: } 075^{\circ} 29^{\prime} 794^{\prime \prime}\end{array}$ & 36 \\
\hline Ciénagade Oro & Sinú medio & Las Palmas & $\begin{array}{l}\text { Longitud:0850’49”, } \\
\text { Latitud: } 075^{\circ} 35^{\prime} 43,5^{\prime \prime}\end{array}$ & 18 \\
\hline Chimá & Bajo Sinú & Galilea & 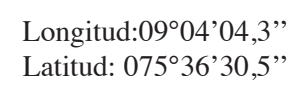 & 18 \\
\hline
\end{tabular}

${ }^{1}$ Número total de ovinos identificados por unidad productiva para la producción de carne / Total number of sheep identified per productive unit for meat production.

\section{Evaluación técnica de la producción de carne de corderos}

Para los análisis técnicos de los sistemas de producción se tomó como unidad de análisis la información de cada unidad productiva, en las variables i) tamaño de la unidad productiva y característica de animales, ii) finalidad del sistema de producción y iii) manejo y desempeño productivo de ovinos en la fase de levante-ceba.

Para evaluar el desempeño de los corderos destinados a la producción de carne, se registró la información del manejo que los productores tradicionalmente realizaron en las cuatro fincas seleccionadas (Cuadro 1). La población animal correspondió a 108 ovinos de pelo colombiano (OPC) en fases de levante-ceba, de edad $\leq 12$ meses, con peso inicial de $18 \pm 3,0 \mathrm{~kg}$, condición corporal de 3,0 puntos. 
Cada veintiocho días se visitaron las unidades productivas para realizar las evaluaciones de la ganancia de peso, condición corporal y ganancia de peso acumulada $\left(\mathrm{g} \mathrm{día}^{-1}\right)$ por cada animal; esta última, se estimó mediante la siguiente fórmula: GDP= $(\mathrm{P}$ final-P inicial $) /$ número de días. Para el pesaje se utilizó balanza de gancho, la condición corporal se evaluó en escala de 1 a 5 de acuerdo con Thompson y Meyer (1994).

\section{Análisis económicos y financieros de los sistemas de producción}

Para el desarrollo de la evaluación económica y financiera de los sistemas de producción, se registró la información de los costos e ingresos de un sistema de producción semi-intensivo que constituye la línea de base de esta investigación, la metodología de los análisis económicos, estuvo apoyada en experiencias previas de estudios realizados por Hernández et al. (2014), debido a ello, la evaluación se realizó teniendo en cuenta los coeficientes técnicos de la producción ovina (manejo del ciclo productivo y tasa de descarte de los animales) como: ganancia de peso, producción de carne y, factor de conversión de kg de carne de animal vivo a $\mathrm{kg}$ de carne en canal, con base en estos se calcularon los indicadores de retorno para medir la bondad económica y financiera de estos sistemas de producción. Posteriormente, se elaboraron los patrones de costos y se calcularon los indicadores de retorno del sistema de producción manejado convencionalmente.

\section{Indicadores económicos del estudio}

- Ingresos brutos: se definen como las cantidades producidas en el mercado nacional, se obtiene multiplicando el peso en pie de ovinos vendidos por el precio de venta del kilogramo (USD $\$ 1,16 \mathrm{~kg}^{-1}$ ).

- Ingreso: es la cantidad de dinero que obtiene el productor por la venta de la carne de ovino expresado en $\mathrm{kg}$ animal $^{-1}$ ) en el mercado a los precios vigentes. En este sentido, el ingreso de la producción es equivalente al valor de la producción final.

- Costos: representa las salidas de dinero por concepto de compra de insumos para el proyecto. Pueden ser fijos o variables. En estos se incluye: mano de obra, insumos, equipos, alimentación y los indirectos como administración, impuestos y costos financieros.

- Costos fijos: son los que no guardan una relación directa con las cantidades producidas y permanecen constates a lo largo del periodo productivo. Por ejemplo, administración, impuestos y costos financieros.

- Costos variables: son aquellos que están en relación directa con las cantidades producidas, aumentan cuando aumenta la producción y disminuyen cuando esta también disminuye. Por ejemplo, mano de obra, insumos, equipos y alimentación.

- Inversiones: se diferenciaron de los costos y constituyeron infraestructura, reparaciones y arreglo de senderos.

- Ingreso neto: es la diferencia entre el ingreso bruto y el costo total. Indica si hay ganancia o pérdida (ecuación 1):

$$
I N=I B-C T
$$

- Rentabilidad: es la relación entre el ingreso neto y el costo total, muestra los retornos o rendimientos de la inversión (ecuación 2):

$$
\operatorname{Re} n t=\frac{I B-C T}{C T} * 100
$$

- $\quad$ Punto de equilibrio (Pe): indica las cantidades máximas de producto que deben producirse para equilibrar los ingresos con los costos.

Para expresar el comportamiento en el tiempo, se usó el concepto del valor del dinero en el tiempo, y se hicieron cálculos de flujos de caja considerando un horizonte de siete años, donde se consideró un periodo cero 
que fue donde se asume se hicieron las inversiones y no genera ingresos, estos tienen lugar a partir del año uno. Igualmente se consideró un costo de oportunidad del $12 \%$ con el cual se compararon los retornos de la inversión. En este caso se usaron los siguientes indicadores:

\section{Evaluación financiera}

Relación beneficio costo (B/C): es el cociente de los ingresos y los costos totales actualizados, obtenidos a partir de la tasa de oportunidad seleccionada (12\%) (ecuación 3):

$$
B / C=\frac{\text { VPingresos } /(1+i)^{n}}{V P \cos t o s /(1+i)^{n}}
$$

Este indicador determina cuántos dólares se obtienen de beneficios por cada unidad monetaria invertida, pero llevado al valor presente, descontados, en ambos casos, a la tasa de oportunidad del inversionista (12\%). Es decir, que para que el proyecto resulte ser factible financieramente la relación beneficio costo debe ser mayor que 1 .

Valor actual neto (VAN): es la suma de las diferencias actualizadas de los ingresos y costos durante los años de vida útil del proyecto. Indica la ganancia neta generada por el proyecto descontados todos los costos del mismo (ecuación 4):

$$
V A N=\sum_{t=0}^{n} \frac{(I B-C T)}{(1+i)^{n}}
$$

Tasa interna de retorno (TIR): se define como la tasa de interés que hace equivalentes los ingresos recibidos y los pagos efectuados durante el tiempo que dure el proyecto de inversión.

El cálculo de la TIR se hace con el empleo de diferentes tasas, hasta lograr una en que el VAN sea igual a cero; o encontrando dos tasas, una que genere un VAN2 negativo que se denominará i2 y otra que genere un VAN1 positivo que se llamará i1 (ecuación 5):

$$
T I R=i_{2}-\operatorname{VAN}_{2}\left\{\left(i_{2}-i_{1}\right) /\left(\operatorname{VAN}_{2}-V A N_{1}\right)\right\}
$$

\section{Cálculos de los márgenes de comercialización}

Como complemento del análisis económico se tuvo en cuenta los cambios en los precios cuando el producto pasa de un eslabón de la cadena productiva a otro, en este sentido se consideró el cambio cuando pasa del productor al intermediario y al consumidor final, por tanto, se aplicó el concepto de margen.

Con la información obtenida del productor primario en cada unidad productiva y los precios del mercado, se calcularon los márgenes de comercialización de la carne de ovinos: margen bruto de comercialización (MBC), la participación directa del productor (PDP) y el margen neto de comercialización (MNC). De acuerdo con Espinosa et al. (2008), el MBC se calcula como la diferencia entre el precio pagado por el consumidor y el que recibe el productor (ecuación 6).

$$
\mathrm{MBC}=\frac{\text { PrecioConsumidor }- \text { Pr ecio } \text { Productor }}{\text { Pr ecioConsumidor }} * 100
$$


Igualmente se calculó la participación directa del productor (PDP): indica la porción que el productor recibe por lo que paga el consumidor (ecuación 7).

$$
\mathrm{PDP}=100-\mathrm{MBC}
$$

Margen neto de comercialización (MNC): este indicador muestra la proporción del precio que paga el consumidor, son utilidades para el intermediario (ecuación 8).

$$
\mathrm{MNC}=\frac{\text { MárgenBruto }- \text { CostosdeMercadeo }}{\text { Pr ecioConsumidor }}
$$

Se trata de estimar las diferencias de los precios que le pagan al productor por su producto en este caso ovino en pie, y los que paga el consumidor por el producto final en este caso la carne en canal.

\section{Análisis estadístico}

Los resultados entre los indicadores económicos y financieros de los sistemas de producción e indicadores del desempeño productivo animal, relacionados con la ganancia de peso y condición corporal, fueron analizados mediante estadística descriptiva, estimando la media, el desvío estándar, coeficiente de variación y rango, para ello se usó el programa estadístico SPSS versión 15.0 (SPSS, 2007). Se realizó un análisis de varianza (ANOVA) para evaluar las ganancias de pesos diaria de los corderos. La comparación de medias se realizó mediante la prueba LSD de Fisher $(\mathrm{p} \leq 0,05)$.

\section{Resultados}

Los resultados de las características biofísicas relacionadas con el tamaño (área de producción), altura, número de animales y finalidad de las fincas del estudio dedicadas a la ovinocultura se describen en la Figura 1.

Se encontró que las finalidades de las unidades productivas del presente trabajo estuvieron orientadas a las siguientes actividades: i) el $90 \%$ de los productores mantenían el ciclo de producción para la venta de corderos de engorde para consumo local y comercialización de carne de animales; el tipo de producción correspondía a la venta de ovinos entre 12 y 16 meses edad con pesos entre 23 y $30 \mathrm{~kg}$; ii) el $10 \%$ de los productores realizaba la venta de ovinos mejorados genéticamente de crías para la repoblación del rebaño. Las razas de ovinos encontrados en las unidades productivas correspondían en un $100 \%$ a ovinos de pelo criollo (OPC) y sus cruces con las razas, Kathadin, Santa Inés, Dorper, Pelibuey. Las cuales han sido consideradas como razas muy adaptadas a las condiciones de alta humedad y temperatura, características de la Región Caribe de Colombia, con resistencia a enfermedades.

\section{Manejo animal y desempeño productivo}

En las unidades productivas estudiadas, el $80 \%$ de los ovinos eran mantenidas en manejo semi intensivo, basado en el pastoreo diurno y el confinamiento nocturno en apriscos adecuados con comederos y bebederos, permitiendo un pastoreo de $10 \mathrm{~h}$ al día. Respecto a la alimentación animal, todos los productores realizaban suplementación mineral (sal) de manera permanente. El $60 \%$ de los productores utilizaba suplementación alimenticia únicamente en la época seca del año. Las especies de pastos que se encontraban en un $64 \%$ de las 


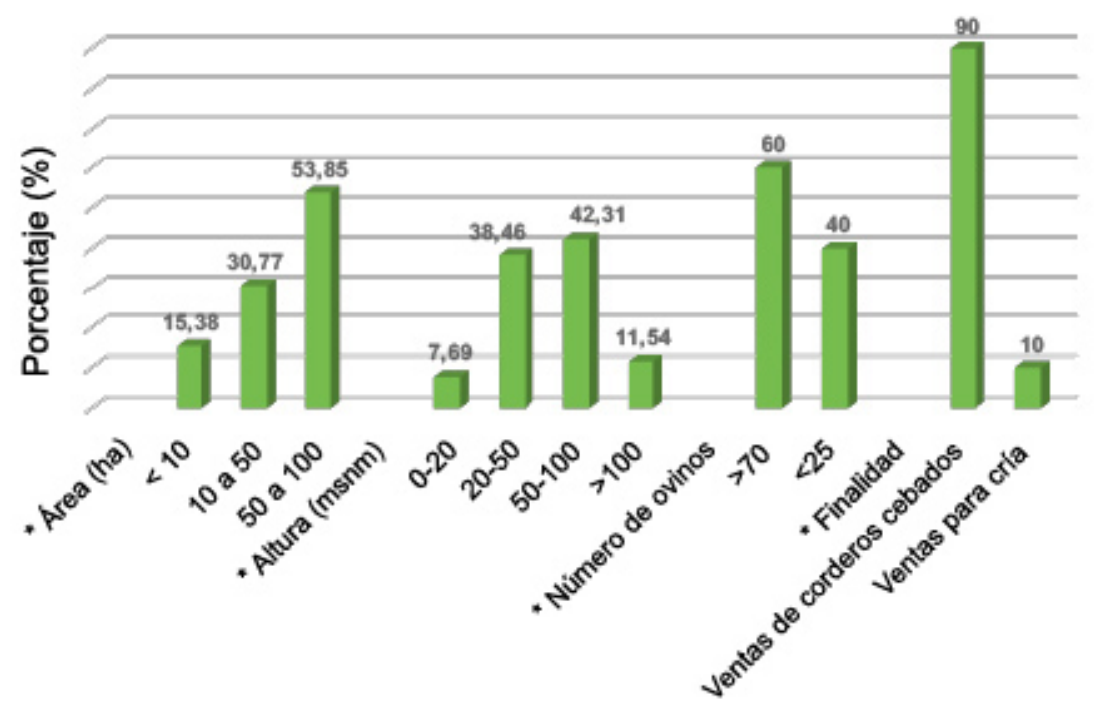

Figura 1. Características biofísicas de los sistemas de producción de ovinos. Córdoba, Colombia. 2018.

Figure 1. Biophysical characteristics of sheep production systems. Cordoba, Colombia. 2018.

UP correspondía a las especies colosuana (Bothriocloa pertusa), donde un $19 \%$ utiliza pasto colosoana en asocio con angleton (Dichantium aristatum), $14 \%$ utilizan pasto estrella (Cynodom nlenfuemsis) y solo el $3 \%$ de los productores mantenía en pasto guinea (Megathyrsus maximus), en las unidades productivas el sistema de pastoreo continuo es predominante.

La evaluación de las ganancias de peso diario (GDP) de corderos en fases de levante-ceba se realizó durante 112 días, en cuatro periodos de 28 días. Se encontró que las ganancias de peso de los animales en las cuatro

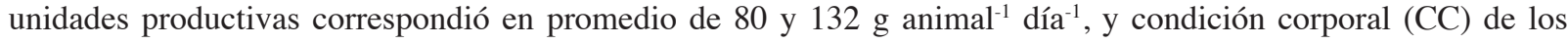
animales al beneficio evaluado, correspondió en promedio a 3,4 de CC, lo que correspondió con animales de $31,71 \pm 3,2 \mathrm{~kg}$ promedio . El peso promedio menor presentó en la unidad productiva Galilea con $27,72 \pm 3,3$ y una CC

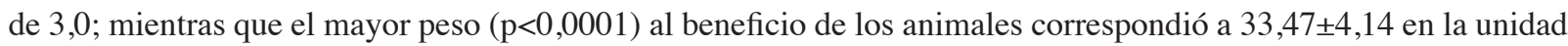
productiva Campanario, animales con una condición corporal media al beneficio de 3,6 ( $\mathrm{p}=0,0063$ ) (Cuadro 2).

\section{Evaluación económica y financiera}

La información resumida de costos obtenidas del componente biofísico de cuatro unidades productivas de ovinos en forma convencional en el departamento de Córdoba se presenta en el Cuadro 3. La producción de carne fue de $18450 \mathrm{~kg}$ entre las cuatro fincas, y un valor de USD \$1,55 por $\mathrm{kg}$ de animal vivo en pie con un peso promedio al beneficio, que entre las cuatro unidades productivas fue de $31,7 \pm 3,2 \mathrm{~kg}^{\text {animal }}{ }^{-1}$.

Los datos permitieron ver diferencias en el costo unitario de producción, donde la unidad productiva Galilea fue la que produjo el $\mathrm{kg}$ de carne a un costo más bajo (USD \$ $0,9 \mathrm{~kg}^{-1}$ ) y el mayor ingreso neto con USD \$1579; sin embargo, no se apreciaron diferencias marcadas entre las unidades productiva analizadas, las cuales presentaron un promedio de USD\$1,04 $\mathrm{kg}^{-1}$, con variaciones muy bajas de acuerdo con el coeficiente de variación del $21 \%$.

Con la información obtenida del productor primario en la unidad productiva, comercializador intermediario y supermercado, se calcularon los márgenes de comercialización de la carne de ovinos: margen bruto de 
Cuadro 2. Promedio de la ganancia de peso diario (GPD) y condición corporal en corderos de las cuatro unidades productiva (UP) hasta la ceba (peso final). Córdoba, Colombia. 2018.

Table 2. Average daily weight gain (DWG) and body condition in lambs from the four productive units (farms) to the fattening phase (final weight). Cordoba, Colombia. 2018.

\begin{tabular}{lccccc}
\hline & \multicolumn{5}{c}{ Fincas } \\
\cline { 2 - 6 } Indicador & Campanario & Esperanza & Las palmas & Galilea & P-Valor \\
\hline${ }^{\mathrm{I}} \mathrm{GPD}\left(\mathrm{g} \mathrm{d}^{-1}\right)$ & $132^{\mathrm{a}}$ & $112^{\mathrm{b}}$ & $100^{\mathrm{b}}$ & $80^{\mathrm{c}}$ & $<0,001^{* * *}$ \\
$\mathrm{CC}$ final & $3,6^{\mathrm{b}}$ & $3,6^{\mathrm{b}}$ & $3,7^{\mathrm{b}}$ & $3,0^{\mathrm{a}}$ & $0,0063^{* *}$ \\
Peso F $(\mathrm{kg})$ & $33,4 \pm 4,1$ & $32,8 \pm 4,4$ & $32,8 \pm 3,6$ & $27,7 \pm 3,3$ &
\end{tabular}

$\mathrm{CC}$ final: condición corporal final, $\mathrm{PF}(\mathrm{kg})$ : peso final. Medias con diferente letra dentro de las filas fueron significativamente diferentes $(\mathrm{p}<0,05)$ / CC final: final body condition, PF $(\mathrm{kg})$ : final weight. Means within a row with different letter were significantly different $(\mathrm{p}<0.05)$.

Cuadro 3. Análisis económico comparativo de costos de producción (USD\$) de cuatro unidades productivas de corderos ubicadas en las sub regiones de San Jorge (Campanario), Sabanas (Esperanza), Medio Sinú (Palmas), Bajo Sinú (Galilea), en el departamento de Córdoba, Colombia. 2018.

Table 3. Comparative economic analysis of production costs (USD\$) of four productive lambs units located in the sub-regions of San Jorge (Campanario), Sabanas (Esperanza), Medio Sinu (Palmas), and Bajo Sinu (Galilea), in the department of Cordoba, Colombia. 2018.

\begin{tabular}{|c|c|c|c|c|c|}
\hline Indicador & Campanario & Esperanza & Palmas & Galilea & Promedio \\
\hline${ }^{1}$ Costo total & 6318 & 4942 & 3362 & 4361 & 4746 \\
\hline Costo unitario & 1,02 & 1,00 & 1,20 & 0,90 & 1,00 \\
\hline Ingreso neto & 1962 & 1658 & 364 & 1579 & 1391 \\
\hline Rentabilidad & 31,0 & 33,6 & 10,83 & 36,2 & 27,9 \\
\hline Punto de equilibrio (Pe) & 4738 & 3706 & 2521 & 3270 & 3559 \\
\hline Porcentaje Pe & 76,3 & 33,6 & 54,2 & 70,3 & 58,6 \\
\hline VPN & 10279 & 6452 & 1222 & 7134 & 6272 \\
\hline TIR & 3,7 & 4,8 & 1,2 & 8,2 & 4,5 \\
\hline CAE & 2047 & 2015 & 1106 & 1739 & 1727 \\
\hline
\end{tabular}

${ }^{1}$ Costos calculados en dólares de Estados Unidos, VPN= valor presente neto, TIR $=$ tasa interna de retorno, CAE $=$ costo anual equivalente $/{ }^{1}$ Costs calculated in United States dollars VPN= net present value, TIR $=$ internal rate of return, CAE= equivalent annual cost.

comercialización (MBC), la participación directa del productor (PDP) y el margen neto de comercialización (MNC). Para estos análisis se partió del hecho que al productor le pagan un precio de USD $\$ 1,16 \mathrm{~kg}^{-1} \mathrm{de}$ animal en pie, este $\mathrm{kg}$ de carne lo vende el intermediario en canal a un precio de USD $\$ 4,33 \mathrm{~kg}^{-1}$, para finalmente venderlo al consumidor a USD $\$ 5,0 \mathrm{~kg}^{-1}$.

El margen bruto de comercialización del 76 \% representó la porción que paga el consumidor para cubrir los gastos y utilidades de la intermediación, es decir que por cada USD $\$ 1,0$ que paga el consumidor, USD\$0,76 son captados por la intermediación. 


\section{Participación directa del productor (PDP)}

La participación directa del productor del $24 \%$ indica que este recibe USD $\$ 0,24$ por cada USD $\$ 1,0$ que paga el consumidor es decir que el $24 \%$ de lo que paga el consumidor es la cantidad que realmente recibe el productor.

El margen neto de comercialización (MNC) fue de 66,6 \%, lo que muestra que por cada USD $\$ 1,0$ que paga el consumidor USD \$0,66 son utilidades que se distribuyen en la cadena de intermediación.

Las variaciones de los diferentes indicadores en las unidades productivas analizadas se aprecian en el Cuadro 4. Bajo el enfoque de usar medidas de dispersión especialmente el coeficiente de variación.

No existieron diferencias muy marcadas de los indicadores de retorno entre las unidades productivas que se analizaron (Cuadro 4).

Cuadro 4. Comparación de indicadores en las diferentes unidades productivas de ovinos mediante las medidas de tendencia central y medidas de dispersión de los indicadores económicos financieros de fincas productoras. Córdoba, Colombia. 2018.

Table 4. Comparison of indicators in the different productive sheep units through the measures of central tendency and measures of dispersion of the financial economic indicators of producer farms in Cordoba, Colombia. 2018.

\begin{tabular}{lcccc}
\hline & Promedio & DS & CV \% & Mediana \\
\hline Costo total & 4745 & 1234 & 26 & 4651 \\
Costo unitario & 1,04 & 0,11 & 11 & 008 \\
Ingreso neto & 1391 & 704 & 51 & 1618 \\
Rentabilidad & 27,9 & 11,6 & 42 & 32,3 \\
Punto de equilibrio & 3559 & 926 & 26 & 3488 \\
Porcentaje Pe. & 58,6 & 19,1 & 33,0 & 62,3 \\
VPN & 6272 & 3756 & 60 & 6793 \\
TIR & 4,5 & 2,9 & 64 & 4,3 \\
CAE & 1727 & 436 & 25 & 1877 \\
\hline
\end{tabular}

${ }^{1}$ Valores monetarios expresados en dólares de Estados Unidos \$USD, VPN: valor presente neto, TIR: tasa interna de retorno, CAE: costo anual equivalente, DS: desviación estándar, CV: coeficiente de variación / ${ }^{1}$ Monetary values expressed in United States dollars USD\$, VPN: net present value, TIR: internal rate of return, CAE: equivalent annual cost. DS: standard deviation, CV: coefficient of variation $(\%)$.

El costo unitario de producción promedio fue de USD $\$ 1,04 \mathrm{~kg}^{-1}$, al compararlo con el costo mínimo de USD \$0,93 en Galilea y un costo máximo por kg de USD \$ 1,2 para el caso de Las Palmas. se presentó una desviación estándar de \$0,11 USD y un coeficiente de variación del $11 \%$, lo que quiere decir que la distribución se acercó mucho a una normal con una mínima dispersión entre valores.

\section{Discusión}

En los sistemas para ovinos para carne del departamento de Córdoba aún se desconoce la dinámica del manejo técnico para la producción de carne de una manera más eficiente. Según Moreno y Grajales (2017), a nivel nacional, en las unidades productivas de ovinos predomina el manejo semi intensivo o extensivo, en consecuencia, los mayores controles productivos se realizan especialmente en los sistemas de producción de genética y de carne con mayor grado de especialización. 
La producción de cualquier especie debería analizar los factores que influyen en el intercambio de un bien (carne ovina). En este estudio, la finalidad principal de los productores que mantienen las explotaciones ovinas en Córdoba, es la producción de ovinos de engorde para el beneficio y comercialización de la carne, estos resultados fueron coherentes con los estudios realizados en el Caribe seco colombiano por Rua et al. (2018), quienes aplicaron encuestas en municipios del Cesar, Colombia, para conocer la línea de base de los sistemas de producción ovinocaprina de esta región, evidenciando que el $90 \%$ de los productores realizaban el ciclo completo del manejo ovino para la producción y comercialización de la carne.

En la mayoría de los casos el sacrificio y la comercialización de carne ovina se realiza de manera informal (Castellanos et al., 2010), lo cual implica que posiblemente la población y producción real de carne de ovinos a nivel del departamento sea el doble (como mínimo) de las estimaciones oficiales. Bajo este contexto algunos investigadores (Espinal et al., 2006) preconizan que la práctica de la ovinocultura es una buena alternativa de producción agropecuaria, principalmente por las posibilidades geográficas colombianas que la hacen viable y las condiciones favorables que presenta el mercado, con una creciente demanda; esta situación convierte a los ovinos en una de las especies con mayores perspectivas de desarrollo en el área pecuaria en el país y una alternativa para la seguridad alimentaria.

En las unidades productivas evaluadas, se reportó que en el $80 \%$ mantienen a los animales de manera semi intensiva, y el desarrollo de la producción ovina se realiza mediante pastoreo extensivo sin prácticas de manejo alimenticio y/o suplementación, solo en la época seca del año el $60 \%$ de los productores realizan suplementación únicamente en la época seca del año. Los sistemas de producción de pequeños rumiantes dependen del clima para la producción de forraje, el cual es la base de su alimentación diaria, por lo que en épocas criticas del año, cuando se presentan inviernos o veranos fuertes y prolongados, se disminuye o anula la disponibilidad de alimento (Grajales et al., 2011). Lo anterior, representa restricciones en el desempeño animal por limitarse la ingestión en cantidad y calidad de nutrientes, que posiblemente no sean suficientes para fomentar el potencial productivo, que se vería reflejado en una mayor ganancia de peso durante las fases de levante y ceba de los animales, hasta su beneficio (Kawas y Huston, 1990; Burns et al, 1991; Mendives, 2007; Nuñez et al., 2007; Kawas, 2008).

En las unidades productivas de este estudio, la mayoría de los productores aún desconocen el manejo agronómico del recurso forrajero, la planificación de las áreas disponibles para el pastoreo animal, así como la composición nutricional de los forrajes para la alimentación y sus beneficios, en términos de disponibilidad de proteína (\% PC) de las variedades de forrajes que predominan en sus unidades productivas, las cuales han sido reportados en estudios realizados en el departamento de Córdoba por Suárez-Paternina et al. (2015), para los pastos Cynodon nlemfuensis (estrella) del 13,56 \% PC y Megathyrsus maximus (mombasa) de 11,22 \% de PC, y por Montoya et al. (2003) para el pasto Dichatium aristatum (angleton) de 7,0 a 11,3\% PC, en época seca y época de lluvia respectivamente.

Una de las mayores ganancias de peso diario (GDP) de ovinos criollos (OPC) en fase de ceba correspondió a $130 \pm 34,6 \mathrm{~g} \mathrm{día}^{-1}$, resultados que coinciden con otros estudios que también han sido realizados en ovinos criollos en fase de ceba para la producción de carne en el departamento de Córdoba (Maza et al., 2016), donde se han

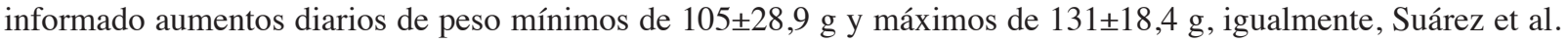
(2018), reportaron valores mínimos de 53,6 g y máximos de 130,5 g. Los anteriores resultados, superan los valores que a nivel nacional se ha registrado como referentes para los ovinos criollos de $108 \mathrm{~g}$ animal $^{-1}$ día $^{-1}$ (Cortés, 2012).

Respecto al peso final para el beneficio de los ovinos, los productores de Córdoba consideraron en promedio de 25 a $30 \mathrm{~kg}$. El promedio de peso al beneficio observado en las unidades productivas de este estudio, correspondió $31,7 \pm 3,2 \mathrm{~kg}$, y los mayores pesos finales para el beneficio de animales del estudio de referencia, se presentaron en las

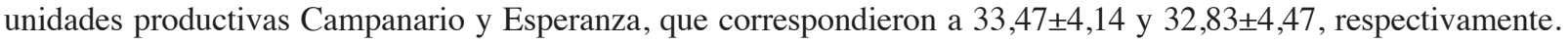
Las mejores ganancias de peso que se observaron en estas unidades productivas, pueden atribuirse a la mayor oferta y calidad de los forrajes disponibles en las unidades. Al respecto, algunos investigadores han evidenciado que en la alimentación de rumiantes en pastoreo, la cantidad de alimento consumido es el principal factor que determina la productividad animal (Allison, 1985; Detmann et al., 2005; Costa et al., 2015) y reconocen que la producción diaria 
de los animales está dada por la combinación de efectos entre la disponibilidad y calidad del alimento, así como el apetito y la genética del animal (Nuñez et al., 2007).

Las diferencias en el costo unitario de producción se atribuyeron a los cambios en las unidades producidas, principalmente por considerar que los costos totales presentaron diferencias relativamente bajas con un coeficiente de variación del $26 \%$. Por otra parte, los indicadores de retorno permitieron concluir que este sistema de producción es sostenible económicamente en todos los casos analizados, en la medida que no se aprecian diferencias marcadas entre las unidades productivas analizadas con un promedio de USD $\$ 1,059 \mathrm{~kg}^{-1}$. Este resultado se compara con otras investigaciones realizadas por Borroto et al. (2011) en la región Ciego de Ávila, Cuba, donde se presentaron cantidades productivas similares a las de este trabajo, con promedio de $32,2 \mathrm{~kg}$ animal ${ }^{-1}$, en tanto que en el presente estudio se

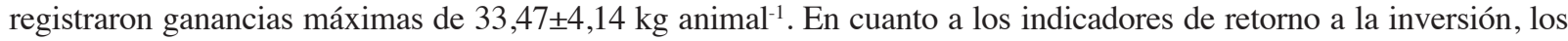
resultados obtenidos fueron más altos que los registrados en Yucatán, México por Hernández et al. (2014). Con estos datos se afirma que, estos sistemas en todas las fincas presentaron ganancia económica por tener un ingreso neto positivo, lo que significa que en todos los casos el valor monetario de los ingresos fue mayor que el de los costos.

Con relación a los márgenes de comercialización se pudo establecer que los resultados encontrados en este estudio fueron muy similares a los de Hernández-Martínez et al. (2013), quienes en el estado de México encontraron que el productor participó con el 17,75\% del precio que pagó el consumidor final, en el caso de este estudio el productor participó con el $24 \%$, esto lo que muestra es que existe inequidad en la distribución de los excedentes a lo largo de la cadena productiva, donde el productor quien es el que más arriesga es el que menor participación tiene.

\section{Conclusiones}

Los sistemas de producción que se evaluaron fueron rentables al juzgarlos por los indicadores de retorno, que en ninguno de los casos los costos superaron los ingresos, debido a los bajos costos de producción y con costos unitarios, lo cual permitió alcanzar tasas de rentabilidad por arriba del $20 \%$ en cada ciclo de producción, esto representa una garantía de la eficiencia del sistema de producción por cuanto la tasa de recuperación del capital es superior al costo de oportunidad del mismo. Para los casos analizados, el mayor beneficiario es el intermediario, ya que le corresponde el $76 \%$ de la diferencia entre el precio de venta al producto y el precio que paga el consumidor.

La evaluación productiva de corderos de las unidades de estudio bajo el manejo semi-intensivo, evidenció que las mayores ganancias de peso $\left(\mathrm{g} \mathrm{d}^{-1}\right)$ durante el periodo de evaluación fueron superiores en los sistemas que establecieron las mejores condiciones de manejo y alimenticias en los animales, con un registro que superó en 33,5\% de la ganancia de peso diaria y representó a la venta de los corderos la mayor relación costo-beneficio, y el ingreso neto positivo para el sistema de producción.

\section{Agradecimientos}

Los autores agradecen el desarrollo de este estudio, parte de la agenda de investigación de la Red de Ganadería

y Especies Menores, a la Corporación Colombiana de Investigación Agropecuaria (Agrosavia), Centro de Investigación Turipaná, y al Ministerio de Agricultura y Desarrollo Rural de Colombia (MADR).

\section{Literatura citada}

Acero-Plazas, V.M. 2014. El bienestar animal en sistemas productivos de ovinos-caprinos en Colombia. Spei Domus. 10(21):5762. doi:10.16925/sp.v10i21.918 
Allison, C.D. 1985. Factors affecting forage intake by range ruminants: a review. J. Range Manag. 38:305-311. doi: $10.2307 / 3899409$

Borroto, Á., C.R. Pérez, C.A Mazorra, C.A. Pérez, y Á.C. Arencibia. 2011. Caracterización socioeconómica y tecnológica de la producción ovina en Ciego de Ávila, región Central de Cuba (Parte I). Pastos y Forrajes 34(2):199-210.

Burns, J.C., K.R. Pond, and D.S. Fisher. 1991. Effect of grass species on grazing steers: II dry matter intake and digesta kinetics. J. Anim. Sci. 69:1199-1204. doi:10.2527/1991.6931199x

Castellanos, J.G., J.C. Rodríguez, W.L. Toro, y C.L. Luengas. 2010. Agenda prospectiva de investigación y desarrollo tecnológico para la cadena productiva cárnica ovino-caprina en Colombia. Ministerio de Agricultura y Desarrollo Rural (MADR), Bogotá, COL.

CIMMYT. 1998. La formulación de recomendaciones a partir de datos agronómicos: Un manual metodológico de evaluación económica. CIMMYT, México D.F., MEX.

Cortés, H. 2012. Situación del recurso ovino y caprino en Colombia. Documento de trabajo elaborado en el marco del plan nacional de acción para la conservación mejoramiento y utilización sostenible de los recursos genéticos animales de Colombia. Bogota, COL. Scribd, USA. https://es.scribd.com/document/363996777/Situacion-Recursos-Ovino-Caprino (consultado 3 ene. 2019).

Costa, N., A.L. Monteiro, A.L.P. Silva, A. de-Moraes, A.F. Giostri, T.S.S. Stivari, S. Gilaverte, T.C. Baldissera, e E.A. Pin. 2015. Considerações sobre a degradação da fibra em forragens tropicais associada com suplementos energéticos ou nitrogenados. Arch. Zootec. 64(R):31-41. doi:10.21071/az.v64i247.504

Detmann, E., M. Paulino, P.R. Cecon, S. Valadares-Filho, J. Zervoudakis, L. Cabral, M. Leão, R. Lana, e N.J. Ponciano. 2005. Níveis de proteína em suplementos para terminação de bovinos em pastejo durante o período de transição seca/águas: consumo voluntário e trânsito de partículas. R. Bras. Zootec. 34:1371-1379. doi:10.1590/S1516-35982005000400035.

Espinal, C.F., H. Martínez, y J.E. Amézquita. 2006. La cadena ovina y caprina en Colombia. Documento de trabajo No. 125. Ministerio de Agricultura y Desarrollo Rural. Agronet, Bogotá, COL. http://bibliotecadigital.agronet.gov.co/ bitstream/11348/3914/1/20078611357_caracterizacion_ovinosycaprinos.pdf (consultado24 oct. 2018).

Espinosa, V.E., G. Rivera, y L.A. García. 2008. Los canales y márgenes de comercialización de la leche cruda producida en sistema familiar (estudio de caso). Vet. Méx. 39(1):1-16.

FAO. 2014. World mapping of animal feeding systems in the dairy sector. FAO, Rome, ITA. http://www.fao.org/3/a-i3913e.pdf (accessed Sep. 8, 2018).

FAOSTAT. 2017. Global statistical yearbook, FAO regional statistical yearbooks. FAO, Rome, ITA. http://www.fao.org/faostat/ en/\#data/QL (accessed Feb. 15, 2019).

Freire, V., D. Agüero, M. Ponce-Crivellaro, M. Vigliocco, y G. Sandoval. 2013. Análisis económico de sistemas productivos ovinos de Córdoba, Argentina. Estudio de casos AgriScientia 30(1):37-47.

Garnier, J.P. 2010. Análisis del mercado mundial de la carne de ovino. Eurocarne 184:115-122.

Grajales, H.A, D.C. Moreno, y E.A. Cárdenas. 2011. Guía técnica de producción ovina y caprina: IV. Aspectos de manejo y control nutricional y alimenticio. International Print Ltda., Bogotá, COL.

Hernández-Martínez, J., M.I. Ortíz-Rivera, S. Rebollar-Rebollar,E. Guzmán-Soria, y F.J. González-Razo. 2013. Comercialización de ovinos de pelo en los municipios de Tejupilco y Amatepec del Estado de México. Agron. Mesoam. 24:195-201. doi:10.15517/am.v24i1.9798

Hernández, C.I., Rejón. A.M., Valencia, H.E, y Araujo. A.L. 2014. Análisis de inversión para la producción de ovinos en el municipio de Tzucacab, Yucatán, México. Rev. Mex. Agroneg. 34:677-687.

Kawas, J.R. 2008. Producción y utilización de bloques multinutrientes como complemento de forrajes de baja calidad para caprinos y ovinos: la experiencia en regiones semiáridas. Tecnol. Ciên. Agropec. 2(3):63-69. 
Kawas, J.R., and J.E. Huston. 1990. Nutrient requirements of hair sheep in tropical and subtropical regions. In: M. Shelton, and E.A. Pereira. editors, Hair sheep production in tropical and sub-tropical regions with reference to northeast Brazil and the countries of the Caribbean, Central America, and South America. Small Ruminant Collaborative Research Support Program, Davis, CA, USA. p. 37.

MADR (Ministerio de Agricultura y Desarrollo Rural). 2018. Crece consumo interno de carne de ovinos y caprinos, gracias al trabajo conjunto entre gobierno y productores. Bogotá, COL. https://www.minagricultura.gov.co/noticias/Paginas/ Crece-consumo-interno-de-carne-de-ovinos-y-caprinos,-gracias-al-trabajo-conjunto-entre-gobierno-y-productores .aspx (consultado 28 nov. 2018).

Maza, L., S. Villa, H. Vergara, M. Bustamante, J. Simanca, A. Alemán, y O. Vergara. 2016. Weight gain and carcass yield in sheep supplemented with multinutrient blocks in transition period dry-rainy season. RECIA 8(1):65-71. doi:10.24188/ recia.v8.n1.2016.224

Mendives, J.A. 2007. Importancia de los ovinos tropicales introducidos al país: Características productivas y reproductivas. Arch. Latinoam. Prod. Anim. 15(1):310-315.

Montoya, J., L. Torregroza, M. Palomino, M. González, H. Cuadrado, S. Reza, y U. Gómez. 2003. Análisis técnico y económico de un modelo de producción de carne en el Valle del Sinú. Rev. MVZ Córdoba 8:265-272. doi:10.21897/rmvz.1051

Moreno, D.C., y H.A. Grajales. 2017. Caracterización de los sistemas de producción ovinos de trópico alto en Colombia: manejo e indicadores productivos y reproductivos. Rev. Fac. Med. Vet. Zootec. 64(3):36-51. doi:10.15446/rfmvz.v64n3.68693

Núñez, A.C., P.R. Mencio, I.D. Renteria, A.S. Solís, y M.L. Ortega. 2007. Influencia de la suplementación sobre la ganancia de peso y calidad de la canal en borregos Dorper/Katahdin. Rev. Cient. UDO Agric. 7(1):245-251.

Rua, C.V., P. Cortés, J.S. Pineda, y L. Rios. 2018. Cadena de valor ovino caprina de la región Caribe seco de Colombia: 1. Diagnóstico de los sistemas de producción ovino caprina del departamento del Cesar. Presentado en: XXVI Reunión de la Asociación Latinoamericana de Producción Animal, y V Simposio Internacional de Producción Animal Guayaquil, 28-31 May. 2018. Ponencia OC06. ECU.

SPSS (Statistical Package for Social Sciences). 2007. Version 15.0 for Windows User's Guide. SPSS Inc., Chicago, IL, USA.

Suárez, E., A. Libardo, A. Wilson, R. Patiño, M. Bustamante, y O. Vergara. 2018. Efecto de la suplementación con semilla de algodón y maíz molido sobre el consumo y el desempeño productivo de ovinos de pelo colombiano. Rev. MVZ Córdoba 23(S):7048-7061. doi:10.21897/rmvz.1426

Suárez-Paternina, E., S. Reza-García, H. Cuadrado-Capella, I. Pastrana-Vargas, M. Espinosa-Carvajal, y S. Mejía-Kerguelén. 2015. Variación en la concentración de sólidos solubles durante el día, en tres pasturas en época seca en el valle medio del río Sinú. Corpoica Cienc. Tecnol. Agropecu. 16:181-188. doi:10.21930/rcta.vol16_num2_art:367

Thompson, J.M., and H.H. Meyer.1994. Body condition scoring of sheep. Oregon Satate University, OR, USA. https://ir.library. oregonstate.edu/concern/administrative_report_or_publications/kk91fk644 (accessed Mar. 9, 2019).

Vega-Pérez, C.A. 2017. Prácticas ganaderas en sistemas de producción de ovinos: desafíos para el mejoramiento de la competitividad del sector en Colombia. Tesis Doc., Universidad Nacional de Colombia, Bogotá, COL.

Zervas, G., and E. Tsiplakou. 2011. The effect of feeding systems on the characteristics of products from small ruminants. Small Ruminant Res. 101:140-149. doi:10.1016/j.smallrumres.2011.09.034 